\title{
Matched asymptotic expansions in financial engineering
}

\author{
Sam Howison* \\ OCIAM and Nomura Centre for Quantitave Finance \\ Mathematical Institute \\ Oxford University
}

May 12, 2005

\begin{abstract}
Modern financial practice depends heavily on mathematics and a correspondingly large theory has grown up to meet this demand. This paper focuses on the use of matched asymptotic expansions in option pricing; it presents illustrations of the approach in 'plain vanilla' option valuation, in valuation using a fast mean-reverting-stochastic volatility model, and in a model for illiquid markets. A tentative framework for matched asymptotic expansions applied directly to stochastic processes of diffusion type is also proposed.
\end{abstract}

Keywords: option pricing, matched asymptotic expansions, financial derivatives, stochastic volatility.

\section{Preface}

It is an honour to have been asked write the 2005 James Lighthill Memorial Paper, and I am most grateful to the Editors of the Journal of Engineering Mathematics for their invitation. When I was a graduate student Lighthill was one of a very small number of colossi of applied mathematics in the UK and I was fortunate to hear a number of talks by him, given in his characteristic style.

I have been asked to write on the subject of mathematical finance, an area in which as far as I am aware Lighthill never worked, although he included a chapter on the subject in [1]. However, one of Lighthill's best-known works is the book Introduction to Fourier Analysis and Generalised Functions [2] which introduced many, many applied mathematicians to the idea of the delta function as a limit of a sequence of 'normal' functions, for example parametrised continuously by a parameter $t$ which may be thought of as time; Lighthill developed the theory of distributions within this framework. This idea sees a natural

*24-29 St Giles, Oxford OX1 3LB. UK, howison@maths.ox.ac.uk 
interpretation in finance, where option prices in the standard Black-Scholes theory demonstrate just such a smoothing effect on singular 'payoff' functions as time to expiry increases; indeed, the 'Gamma' of a vanilla call or put option is exactly equal to the delta function at the expiry date. The theme of this paper, therefore, is to illustrate how the smoothing idea can fruitfully be combined with the techniques of matched asymptotic expansions in a variety of models in finance.

\section{Introduction}

The modern theory of finance, and especially of financial derivatives and financial engineering, depends absolutely on mathematics. Since the subject was kick-started by the work of Black \& Scholes [3] and Merton [4] there has been a very fruitful two-way technology transfer between a range of areas of mathematics and the financial applications, the principal mathematical area being applied probability and in particular applied and numerical stochastic analysis. However, the fact that the Black-Scholes methodology leads, via Itô calculus, to partial differential equations, has led many physical applied mathematicians to contribute to the field; this is the impetus behind, for example, the early text [5]. Much of this activity has focused on obtaining exact solutions (which are particularly useful in the financial context) to certain boundary value problems representing the prices of options, or on numerical methods. The purpose of the current article is to illustrate the scope for another technique, asymptotic analysis, to make a useful contribution to the solution of financial problems, with a particular emphasis on the use of matched asymptotic expansions.

We begin in Section 3 with some simple examples of matched asymptotic expansions applied to vanilla options close to their expiry date. In Section 4 we consider a nonlinear model arising from the study of illiquid markets, and we contrast the smoothing of the payoff singularity by nonlinear diffusion with the effect of linear diffusion demonstrated in Section 3. In Section 5 we turn to a model of fast mean-reverting stochastic volatility and we show how to construct the boundary layer near expiry for European options. Finally in Section 6 we speculate on the possibility of applying the methodology of matched asymptotic expansions directly to stochastic processes, without going via the associated Kolmogorov partial differential equations.

Before proceeding, we recall the standard Black-Scholes formulation of derivatives pricing (see [5]). We consider options on an asset whose price $S_{t}$ is modelled as a function ${ }^{1}$ of time $t$ by the stochastic differential equation

$$
\frac{\mathrm{d} S_{t}}{S_{t}}=\mu \mathrm{d} t+\sigma \mathrm{d} W_{t},
$$

in which $\mathrm{d} W_{t}$ is the increment of a standard Brownian Motion and $\mu$ and $\sigma$ are, respectively, the drift and volatility of the asset, taken to be constant, except in

\footnotetext{
${ }^{1}$ We include the customary subscript $t$ on variables such as $S_{t}$ when we wish to emphasise the time-evolution of the process. In other contexts it may be omitted.
} 
Section 5 where $\sigma$ is also taken to be stochastic. Given an option whose price $V(S, t)$ depends only on $S$ and $t$, application of Itô's formula to the evolution of a hedged portfolio $\Pi=V-\Delta S$, consisting of one option and $-\Delta$ of the asset, shows that the risk in the option is perfectly correlated with that of the asset and that the choice

$$
\Delta=\frac{\partial V}{\partial S}
$$

renders the portfolio instantaneously risk-free. In the absence of arbitrage and transaction costs, the portfolio must then earn the risk-free rate $r$, also taken to be constant, so that $d \Pi=r \Pi \mathrm{d} t$; in the absence of dividends this leads to the Black-Scholes equation

$$
\frac{\partial V}{\partial t}+\frac{1}{2} \sigma^{2} S^{2} \frac{\partial^{2} V}{\partial S^{2}}+r S \frac{\partial V}{\partial S}-r V=0
$$

In the simplest cases this backward parabolic equation is to be solved with a terminal condition $V(S, T)=P(S)$ where $P(S)$ is the payoff received at the expiry date $t=T$, and in the absence of barriers $S$ ranges from 0 to $\infty$. The simplest ('plain vanilla') options are call and put options, whose payoffs are $\max (S-K, 0)$ and $\max (K-S, 0)$ respectively; they represent the terminal value of an option to buy (call) or sell (put) the asset at time $T$ for the fixed price $K$, called the strike price.

An alternative view of the hedging strategy is that it entails pricing with respect to a probability measure $\mathbb{Q}$ that is risk-neutral, rather than the objective (observed) measure $\mathbb{P}$ associated with the stochastic differential equation for the asset price cited above. That is, for pricing purposes, the asset is assumed to follow

$$
\frac{\mathrm{d} S_{t}}{S_{t}}=r \mathrm{~d} t+\sigma \mathrm{d} W_{t},
$$

where $r$ is the risk-free rate, and then the value of the option is

$$
V\left(S_{t}, t\right)=\mathbb{E}^{\mathbb{Q}}\left[P\left(S_{T}\right) \mid S_{t}\right]
$$

By the Feynman-Kac formulas, this expectation is equivalent to the solution of the Black-Scholes equation.

As an aside, we comment that in practice the hedging strategy above is impractical and in particular it is impossible to hedge continuously in time: even if the Wiener process were an exact description of asset prices rather than a good approximation, it would still be impossible to trade either instantaneously or at each $t$. For this reason the hedge parameter termed Gamma,

$$
\Gamma=\frac{\partial^{2} V}{\partial S^{2}}
$$

is of enormous practical importance as it is a measure of the risk incurred in rehedging at non-infinitesimal time intervals. To see this, suppose that a portfolio $\Pi=V-\Delta_{t} S$, where $\Delta_{t}=\partial V / \partial S$ evaluated at $\left(S_{t}, t\right)$, is perfectly hedged at time $t$, and that no trading takes place over the interval $(t, t+\delta t)$. 
Using Taylor's theorem, the change in the portfolio over this interval is $\delta \Pi=$ $\delta V-\Delta_{t} \delta S$ which, in the infinitesimal limit where $\delta t$ becomes $\mathrm{d} t$, is equal to the risk-free return $r \Pi \mathrm{d} t$. Over the non-infinitesimal interval the hedging error (the difference between the return on the portfolio and the risk-free rate) is

$$
\begin{aligned}
\delta \Pi-\Pi\left(e^{r \delta t}-1\right) & =\frac{\partial V}{\partial t} \delta t+\frac{1}{2} \frac{\partial^{2} V}{\partial S^{2}} \delta S^{2}-r\left(V-\frac{\partial V}{\partial S} \delta S\right) \delta t+o(\delta t) \\
& =\frac{1}{2} \sigma^{2} S_{t}^{2}\left(\delta W^{2}-\delta t\right) \frac{\partial^{2} V}{\partial S^{2}}+o(\delta t)
\end{aligned}
$$

where we have used the Black-Scholes equation and the fact to this order $\delta S^{2}=$ $\sigma^{2} S^{2} \delta W^{2}$, the random variable $\delta W$, whose distribution is $N(0, \delta t)$, being the small change in the Wiener process $W$. The error is seen to be proportional to the random variable $\delta W^{2}-\delta t$, whose expectation is zero, multiplied by the option's Gamma; the calculation is equivalent to comparing tangent-plane and quadratic approximations to the value surface. For a call option, we have

$$
\Gamma(S, T)=\frac{\mathrm{d}^{2}}{\mathrm{~d} S^{2}} \max (S-K, 0)=\delta(S-K)
$$

where $\delta(\cdot)$ is the delta function, and the same for a put. Bearing in mind that for a call option without dividends $[\Delta]_{S=0}^{S=\infty}=1$ for all $t$, so that $\int_{0}^{\infty} \Gamma(S, t) \mathrm{d} S=1$, we see that as $t \rightarrow T$ the Gamma of such an option is an approximation of the delta function in the spirit of [2].

\section{$3 \quad$ Vanilla options near expiry}

Our first example is the behaviour near expiry of a call option (or, by put-call parity, a put option) in the standard Black-Scholes model. Here the payoff is $P(S)=\max (S-K, 0)$ and there is a famous explicit formula for the option value,

$$
V(S, t)=S N\left(d_{+}\right)-K e^{-r(T-t)} N\left(d_{-}\right),
$$

where

$$
d_{ \pm}=\frac{\log (S / K)+\left(r \pm \frac{1}{2} \sigma^{2}(T-t)\right)}{\sigma \sqrt{T-t}}
$$

and

$$
N(d)=\frac{1}{\sqrt{2 \pi}} \int_{-\infty}^{d} e^{-s^{2} / 2} \mathrm{~d} s
$$

is the standard Normal cumulative density function. For small time, we can derive an approximation to this formula as follows.

First we make some preliminary scalings: we measure time backwards from expiry and scale it with $\sigma^{2}$, writing $t=T-t^{\prime} / \sigma^{2}$. The Black-Scholes equation becomes

$$
\frac{\partial V}{\partial t^{\prime}}=\frac{1}{2} S^{2} \frac{\partial^{2} V}{\partial S^{2}}+\alpha S \frac{\partial V}{\partial S}-\alpha V
$$


where $\alpha=r / \sigma^{2}$ is dimensionless (at this stage, $V$ and $S$ are still dimensional).

Now suppose that $\alpha=O(1)$, but that scaled time is small, so that

$$
t^{\prime}=\epsilon^{2} \tau
$$

where $0<\epsilon \ll 1$. The Black-Scholes equation is now

$$
\frac{1}{\epsilon^{2}} \frac{\partial V}{\partial \tau}=\frac{1}{2} S^{2} \frac{\partial^{2} V}{\partial S^{2}}+\alpha S \frac{\partial V}{\partial S}-\alpha V
$$

If the spot is far from the strike, we have a regular outer expansion

$$
V(S, \tau) \sim V_{0}(S, \tau)+\epsilon^{2} V_{1}(S, \tau)+\cdots
$$

where

$$
\frac{\partial V_{0}}{\partial \tau}=0, \quad \frac{\partial V_{1}}{\partial \tau}=\alpha\left(S \frac{\partial V_{0}}{\partial S}-V_{0}\right)
$$

and so on, which with the final condition gives

$$
V_{0}+\epsilon^{2} V_{1}=\left\{\begin{array}{lll}
S-K\left(1-\epsilon^{2} \alpha \tau\right) & S-K \gg \epsilon K, & \text { far above the strike, } \\
0 & K-S \gg \epsilon K, & \text { far below the strike. }
\end{array}\right.
$$

This is just the first two terms in the small time expansion (in unscaled variables) of the function

$$
\begin{cases}S-K e^{-r(T-t)} & S>K e^{-r(T-t)}, \\ 0 & S<K e^{-r(T-t)},\end{cases}
$$

whose components are the value of the forward contract in which the option holder is compelled to buy the asset, corresponding to certain exercise, and zero, corresponding to no exercise.

However, as remarked earlier we expect large Gamma near the strike, and hence the second $S$-derivative term cannot be ignored. We deal with this by rescaling near the strike, introducing an inner variable

$$
S=K(1+\epsilon x)
$$

and at the same time rescaling

$$
V(S, \tau)=\epsilon K v(x, \tau) .
$$

The Black-Scholes equation becomes the non-dimensional equation

$$
\frac{1}{\epsilon^{2}} \frac{\partial v}{\partial \tau}=\frac{1}{2 \epsilon^{2}}(1+\epsilon x)^{2} \frac{\partial^{2} v}{\partial x^{2}}+\frac{\alpha}{\epsilon}(1+\epsilon x) \frac{\partial v}{\partial x}-\alpha v,
$$

and the payoff is

$$
v(x, 0)=\epsilon \max (x, 0) .
$$

Having calculated the solution to this inner problem, we will match it with the outer solution. 
We now expand

$$
v(x, \tau ; \epsilon) \sim v_{0}(x, \tau)+\epsilon v_{1}(x, \tau)+O\left(\epsilon^{2}\right) .
$$

Collecting together terms of $O(1)$, the problem for $v_{0}$ is

$$
\frac{\partial v_{0}}{\partial \tau}=\frac{1}{2} \frac{\partial^{2} v_{0}}{\partial x^{2}}, \quad v_{0}(x, 0)=\max (x, 0) .
$$

The conditions at $x= \pm \infty$ for this equation are, consistently with the payoff,

$$
v(x, \tau) \sim x, \quad x \rightarrow+\infty, \quad v(x, \tau) \rightarrow 0, \quad x \rightarrow-\infty .
$$

They can also be obtained by matching, using the Van Dyke rule. This is accomplished by first writing the one-term outer expansion $V_{0}(S, \tau)$ in terms of the inner variables and then expanding to one term in $\epsilon$; the resulting expression is the large- $x$ behaviour of the one-term inner expansion $v_{0}(x, \tau)$.

This inner problem is much simpler than the original problem, and it has a similarity solution

$$
v_{0}(x, \tau)=\sqrt{\tau} f(x / \sqrt{\tau})
$$

where, with $x / \sqrt{\tau}=\xi$,

$$
f^{\prime \prime}+\xi f^{\prime}-f=0,
$$

and with the boundary conditions

$$
f \rightarrow 0 \quad \text { as } \xi \rightarrow-\infty, \quad f \sim \xi \quad \text { as } \xi \rightarrow \infty .
$$

The solution is readily found (it is convenient to differentiate the equation for $f$ first) to be

$$
v_{0}(x, \tau)=x N(x / \sqrt{\tau})+\sqrt{\tau} n(x / \sqrt{\tau})
$$

where $N(\cdot)$ is as above and $n(\cdot)$ is its derivative, $e^{-x^{2} / 2} / \sqrt{2 \pi}$.

The approximation just found is valid in the inner region, while in the outer region we have the outer expansion found earlier. In more complicated problems, one can often find a uniformly valid expansion, holding in both inner and outer regions, by calculating 'outer + inner - common', in which 'outer' and 'inner' are the expansions already found and 'common' is the intermediate limiting behaviour of these expansions used in matching (i.e, the large- $x$ behaviour of the inner expansion or the small $|S-K|$ behaviour of the outer expansion). In our case the outer expansion is so simple that it and the common expansion coincide, and so the inner expansion is in fact uniformly valid and can be used as an approximation for all $S$ and small $t^{\prime}$. In these original variables, this expression is

$$
V(S, t) \sim(S-K) N\left(\frac{S / K-1}{\sigma \sqrt{T-t}}\right)+\sigma \sqrt{T-t} K n\left(\frac{S / K-1}{\sigma \sqrt{T-t}}\right) .
$$

Note that the parameter $\epsilon$, which is artificial, does not appear in this expression. 
It is not hard to proceed further, to find the two-term inner expansion $v_{0}+$ $\epsilon v_{1}$. The problem for $v_{1}$ is

$$
\frac{\partial v_{1}}{\partial \tau}-\frac{1}{2} \frac{\partial^{2} v_{1}}{\partial x^{2}}=x \frac{\partial^{2} v_{0}}{\partial x^{2}}+\alpha \frac{\partial v_{0}}{\partial x}, \quad v_{1}(x, 0)=0 .
$$

As $v_{0}$ satisfies the diffusion equation, a particular solution is readily found ${ }^{2}$ and, as it vanishes at $\tau=0$ it is the solution we need:

$$
\begin{aligned}
v_{1}(x, \tau) & =x \tau \frac{\partial^{2} v_{0}}{\partial x^{2}}+\frac{1}{2} \tau^{2} \frac{\partial^{3} v_{0}}{\partial x^{3}}+\alpha \tau \frac{\partial v_{0}}{\partial x} \\
& =\frac{1}{2} x \sqrt{\tau} n(x / \sqrt{\tau})+\alpha \tau N(x / \sqrt{\tau}) .
\end{aligned}
$$

Furthermore, the two-term inner expansion $\epsilon K\left(v_{0}+\epsilon v_{1}\right)$ is again uniformly valid. In the original variables, this expression now reads

$V(S, t) \sim(S-K+r(T-t)) N\left(\frac{S / K-1}{\sigma \sqrt{T-t}}\right)+\sigma \sqrt{T-t}(S+K) n\left(\frac{S / K-1}{\sigma \sqrt{T-t}}\right)$.

It is also straightforward to show that these expressions agree to $O\left(\epsilon^{2}\right)$ with the small-time expansion of the exact solution.

The approximation is remarkably good for practical parameter values, as shown by the example in Figure 1. Even with a time to expiry of 1 year, for which with $\sigma=0.3$ we have $\sigma^{2} T=0.09$, the maximum relative pricing error is about $2.5 \%$; furthermore the approximate expression can be differentiated to calculate the 'Greeks' (the industry term for $\Delta, \Gamma$ and other partial derivatives of the option price, needed for hedging) as well as the price itself.

As a further test of the accuracy of the approximation, we calculate the error divided by $K\left(e^{-r(T-t)}-(1-r(T-t))\right)$ and, as shown in Figure 2, this is always bounded and tends to 1 as $S \rightarrow \infty$; one expects the errors associated with approximating the discounting to be $O\left((r(T-t))^{2}\right)$ which is small for realistic values, and the figure confirms that the errors associated with the second derivative (volatility) term are not significantly bigger than those arising from approximate discounting.

In this particular example, the outer expansion is simply the outer expansion of the inner problem and the error incurred in simply using the inner expansion is, in fact, exponentially small in $\sigma^{2}(T-t)$; in effect, the inner solution is an expansion of the exact solution in terms of this quantity. If the payoff had been more complicated then the outer expansion would have been non-trivial, and another example in which the method yields non-trivial results is described in the following section.; for more complicated illustrations of the potential of the method in a Black-Scholes setting, see [6].

\footnotetext{
${ }^{2}$ We use the following: (i) if $u_{\tau}-\frac{1}{2} u_{x x}=0$ and $v_{\tau}-\frac{1}{2} v_{x x}=u$, then a particular solution is $\tau u$; (ii) if $u$ is as above and $v_{\tau}-\frac{1}{2} v_{x x}=x u$, then a particular solution is $v=x \tau u+\frac{1}{2} \tau^{2} u_{x}$; (iii) $N^{\prime \prime}(\xi)+\xi N^{\prime}(\xi)=0$.
} 


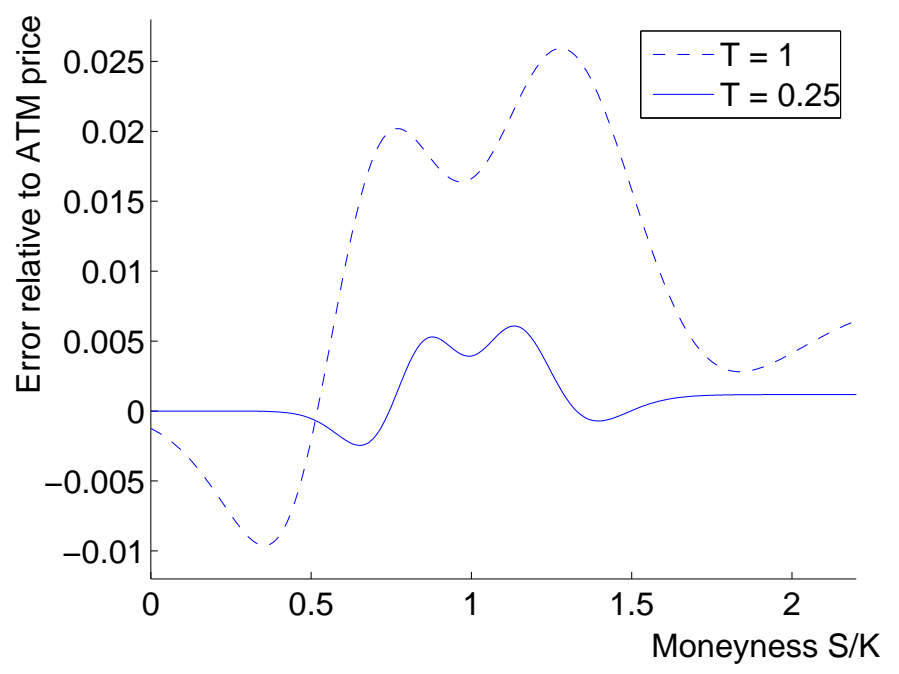

Figure 1: Approximate call value minus exact value, scaled with the at-themoney value, as a function of moneyness $S / K$. Time to expiry one year and 0.25 year. Volatility is $\sigma=0.3, r=0.05$.

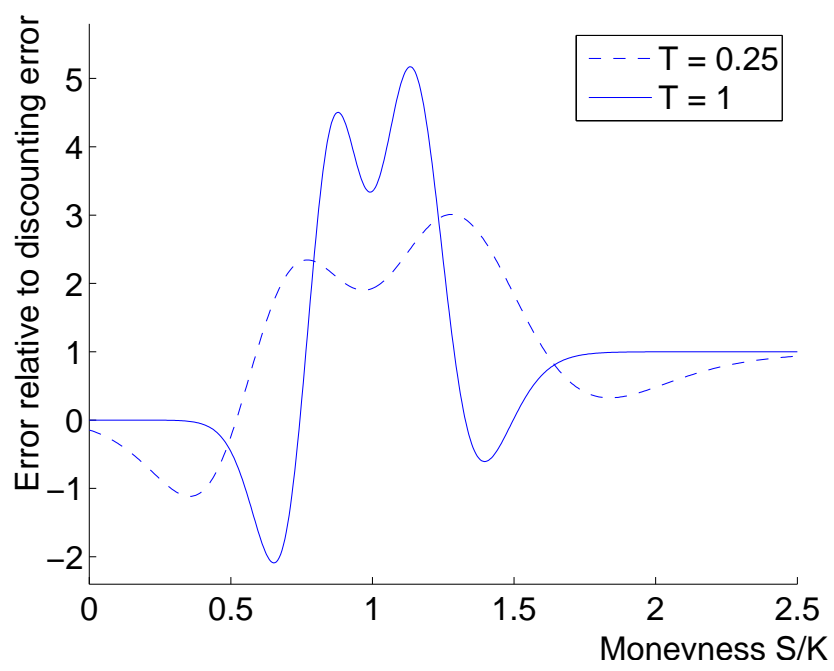

Figure 2: Approximate call value minus exact value, scaled with the discounting error, as a function of moneyness $S / K$. Time to expiry one year and 0.25 year. Volatility is $\sigma=0.3, r=0.05$. 


\subsection{CEV models}

The methodology above can be applied to the constant-elasticity-of-variance $(\mathrm{CEV})$ model in which the Black-Scholes equation is replaced by

$$
\frac{\partial V}{\partial t}+\frac{1}{2} \sigma^{2} S_{0}^{2}\left(\frac{S}{S_{0}}\right)^{\gamma} \frac{\partial^{2} V}{\partial S^{2}}+r S \frac{\partial V}{\partial S}-r V=0 .
$$

The difference from the previous model is that the volatility is now $S$-dependent for $\gamma \neq 2$ (the model is used to represent a 'leverage' effect whereby the impact of a given stochastic change $\mathrm{d} W_{t}$ is assumed to be greater when the asset price is small than when it is large, and $\gamma$ is thus taken to be less than the BlackScholes value of 2). The parameters $S_{0}$ and $\sigma$ are not independent, but writing the equation in this form allows one to compare option prices with the same volatility at a given price level; for example, choosing $S_{0}=K$ gives the same at-the-money volatility for options with strike $K$ as $\gamma$ varies.

It is now much less straightforward to calculate explicit solutions, but the asymptotic procedure is virtually the same as for the case $\gamma=2$, the BlackScholes model. Indeed, the outer expansion is unchanged, while with the scalings previously used, the inner equation becomes

$$
\frac{\partial v}{\partial \tau}=\frac{1}{2} \kappa^{2}(1+\epsilon x)^{\gamma} \frac{\partial^{2} v}{\partial x^{2}}+\epsilon \alpha(1+\epsilon x) \frac{\partial v}{\partial x}-\epsilon^{2} \alpha v,
$$

where $\kappa^{2}=\left(S_{0} / K\right)^{2-\gamma}$, with the same payoff as before. If we rescale time by setting $\tau=\tau_{\gamma} / \kappa^{2}$ and expand to two orders in $\epsilon$, we recover precisely the previous problem for $v_{0}$, so the solution is the same but written in terms of the new time variable $\tau_{\gamma}$. The equation for $v_{1}$ is

$$
\frac{\partial v_{1}}{\partial \tau_{\gamma}}-\frac{1}{2} \frac{\partial^{2} v_{1}}{\partial x^{2}}=\frac{1}{2} \gamma x \frac{\partial^{2} v_{0}}{\partial x^{2}}+\alpha_{\gamma} \tau_{\gamma} \frac{\partial v_{0}}{\partial x}, \quad v_{1}(x, 0)=0 .
$$

where $\alpha_{\gamma}=\alpha / \kappa^{2}$ (note that $\alpha \tau=\alpha_{\gamma} \tau_{\gamma}$ ). We can, therefore, easily adapt the earlier result to write down the inner solution (which is the uniformly valid expansion to this order) as

$$
v_{0}+\epsilon v_{1}=(x+\epsilon \alpha \tau) N(\kappa x / \sqrt{\tau})+\kappa \sqrt{\tau}(1+\epsilon \gamma x / 4) n(\kappa x / \sqrt{\tau}),
$$

which reduces to the previous expression when $\gamma=2$. In original variables, we have the approximation

$$
\begin{aligned}
V(S, t) \sim(S-K+r(T-t)) & N\left(\frac{\kappa(S / K-1)}{\sigma \sqrt{T-t}}\right) \\
& +\kappa \sigma \sqrt{T-t}\left(\frac{\gamma S+(4-\gamma) K}{4}\right) n\left(\frac{\kappa(S / K-1)}{\sigma \sqrt{T-t}}\right) .
\end{aligned}
$$

The accuracy of the approximation is illustrated in Figure 3, which compares the error relative to the at-the-money option value for three different values of $\gamma$. The 'true' value was calculated numerically by an explicit finite difference scheme. The approximation is even better for $\gamma<2$ than it is for the BlackScholes case $\gamma=2$, and appears to improve as $\gamma$ decreases. 


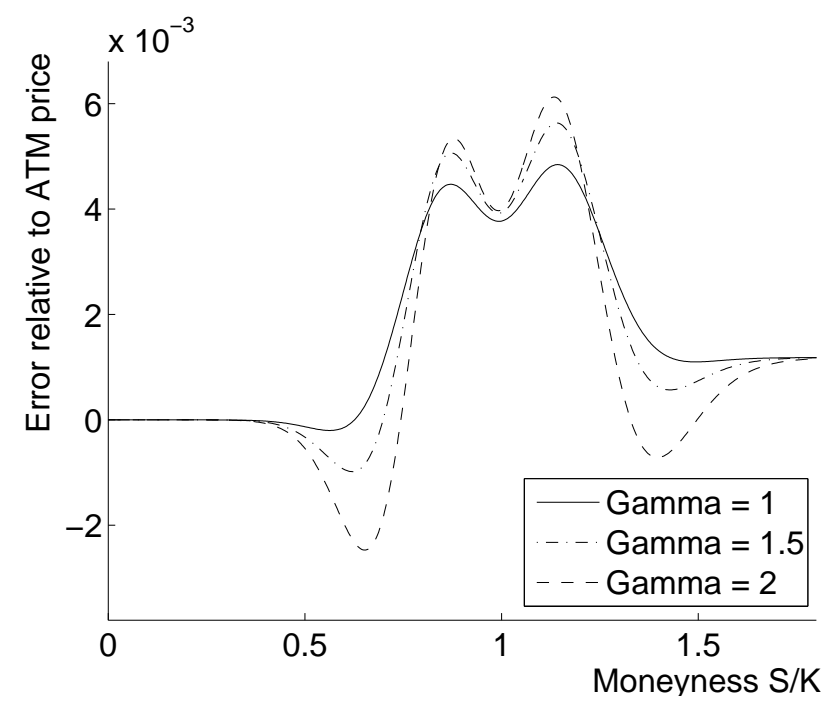

Figure 3: Approximate CEV call value minus numerically calculated value, scaled with the at-the-money numerical value, as a function of moneyness $S / K$. Time to expiry 0.25 year. At-the-money volatility is 0.3 for all options and $r=0.05$.

\section{Vanilla options in an illiquid market}

The standard assumptions of the Black-Scholes model include that of perfect liquidity: it is possible to trade an arbitrary amount of the asset at the price $S_{t}$ without incurring transaction costs and without changing the price by trading. In practice this is simply not true, and in this section we consider an aspect of a model for the effects of illiquidity.

Illiquidity is manifest in three forms in most markets. First, there is a difference, called the bid-offer spread, between the prices for buying and selling even a small amount of the asset. Second, the price paid for trading the asset depends on the amount traded; for example, a trader wishing to buy a large amount of the asset will have to pay a price per unit of the asset that increases with the amount traded, because there is only a limited amount on offer at the lowest offer price (there are usually offers to sell at higher prices as well). This we term the liquidity cost. Third, the action of trading a large amount may itself move the asset price independently of the effect of other random innovations due to external news and the effect of smaller trades. This is termed market impact.

In this section we assume that the bid-offer spread is small and we focus on the effect of liquidity cost and market impact. These have been considered by a number of authors (see $[7,8,9,10,11,12]$ and references therein), and the common thread is that where a 'Black-Scholes equation' can be formulated as a result of a continuous-time trading strategy or a continuous-time approximation to a discrete-time strategy, it is nonlinear (the precise form of the nonlinearity 
depends on the model used). This is not surprising as whenever costs of any sort are incurred, the total value of two positions held separately is in general distinct from the value of the net position, an extreme example being that of a trader who is both long (has bought) and short (has sold) the same contract and whose net exposure is therefore precisely zero and so does not need to be hedged: hedging the two positions separately incurs two sets of costs and thus results in long and short prices whose difference is not zero.

Our interest here is how the nonlinearity affects the valuation of a call or put option near expiry, as in Section 3. As a representative of the class of models used we consider that of [8]. In this model, the price paid to trade a number $N$ of the asset is $S_{t} e^{\lambda N}$, where $S_{t}$ is the asset price before the trade and $\lambda>0$ is a parameter measuring the liquidity of the market; it is always small in practice, but markets with relatively small $\lambda$ are more liquid than those with relatively large $\lambda$. If $N<0$, representing a sale, the price received is less than $S_{t}$, while if $N>0$ the price paid to buy is greater than $S_{t}$. The market impact is represented by assuming that, after the trade, the asset price moves to $S_{t} e^{\beta \lambda N}$, where the parameter $\beta$ measures the market impact, $\beta=0$ representing a trader whose impact on the market is negligible. While the parameter $\lambda$ is typically very small, $\beta$ may be $O(1)$.

With these dynamics superposed on the standard asset price random walk, it is shown in [8] that the model possesses a continuous-time limit in which the value of an option satisfies the nonlinear equation

$$
V_{t}+\frac{1}{2} \sigma^{2} S^{2} V_{S S}+\lambda \sigma^{2} S^{2} V_{S S}^{2}+\frac{1}{2} \lambda^{2} \beta^{2} \sigma^{2} S^{4} V_{S S}^{3}+r S V_{S}-r V=0
$$

(in this section we consistently use subscripts for partial derivatives). Recall that the Gamma, $V_{S S}$, of this option is a delta-function at expiry; following [13], we show how the nonlinear equation for $V$ handles this singularity.

As $\lambda$ is small, we construct a regular outer expansion

$$
V(S, t ; \lambda) \sim V_{0}(S, t)+\lambda V_{1}(S, t)+\lambda^{2} V_{2}(S, t)+O\left(\lambda^{3}\right),
$$

in which

$$
\mathcal{L}_{B S} V_{0}=0, \quad V_{0}(S, T)=P(S)
$$

(we anticipate that the payoff condition should be applied to this function; in this problem the interest is in a small inner region near the strike),

$$
\begin{gathered}
\mathcal{L}_{B S} V_{1}=-\sigma^{2} S^{2} V_{0 S S}^{2}, \quad V_{1}(S, T)=0, \\
\mathcal{L}_{B S} V_{2}=-2 \sigma^{2} S^{3} V_{0 S S} V_{1 S S}-\frac{1}{2} \beta^{2} \sigma^{2} S^{4} V_{0 S S}^{3}, \quad V_{2}(S, T)=0,
\end{gathered}
$$

and so on, where $\mathcal{L}_{B S}$ is the standard Black-Scholes differential operator. Successive terms can be calculated in integral form with the aid of a Green's function or, more likely, numerically (numerical comparisons showing excellent agreement are given in [8]). The expansion is valid provided that higher-order terms remain smaller than lower-order ones, and breakdown first occurs when the magnitudes 
of $V_{0}$ and $\lambda V_{1}$ become comparable. This is for asset values near the strike and for times near expiry, specifically (recalling the results of Section 3 ) for $|(S-K) / K|=O(\lambda)$ and $\sigma^{2}(T-t)=O\left(\lambda^{2}\right)$. We therefore rescale, writing $\sigma^{2}(T-t)=\lambda^{2} \tau$ and $S=K(1+\lambda x)$, and $V(S, t)=\lambda v(x, \tau)$. The leading order term in an expansion in powers of $\lambda$ in the inner region then satisfies

$$
v_{0 \tau}=\frac{1}{2} v_{0 x x}+v_{0 x x}^{2}+\frac{1}{2} \beta^{2} v_{0 x x}^{3}, \quad v_{0}(x, 0)=\max (x, 0) .
$$

This problem is itself intractable but its small and large time behaviour can be calculated. The large-time behaviour is straightforward, as for large times the dominant term on the right-hand side is $\frac{1}{2} v_{0 x x}$, namely a linear diffusion term, and the asymptotic behaviour is that of linear diffusion, matching with the inner limit of the outer solution as discussed in Section 3. The short-time behaviour is, conversely, dominated by the balance between $v_{0 \tau}$ and $\frac{1}{2} \beta^{2} v_{0 x x}^{3}$. We analyse this by first setting $\tau=\epsilon \bar{\tau}, x=\epsilon^{\frac{1}{2}} \bar{x}, v_{0}(x, \tau)=\epsilon^{\frac{1}{2}} \sqrt{2} \bar{v}(\bar{x}, \bar{\tau}) / \beta$, where $\epsilon$ is an artificial small parameter, to find

$$
\bar{v}_{\bar{\tau}}=\bar{v}_{\bar{x} \bar{x}}^{3}+(\sqrt{2} / \beta) \epsilon^{\frac{1}{2}} \bar{v}_{\bar{x} \bar{x}}^{2}+\frac{1}{2} \epsilon \bar{v}_{\bar{x} \bar{x}}, \quad \bar{v}(\bar{x}, 0)=(\beta / \sqrt{2}) \max (\bar{x}, 0) .
$$

By differentiating twice and making the substitution $\bar{v}_{\bar{x} \bar{x}}=u$, the equation for $v$ can be transformed into one of a type familiar in nonlinear diffusion, namely

$$
u_{\bar{\tau}}=\left(u^{3}+(\sqrt{2} / \beta) \epsilon^{\frac{1}{2}} u^{2}+\frac{1}{2} \epsilon u\right)_{\bar{x} \bar{x}}, \quad u(\bar{x}, 0)=(\beta / \sqrt{2}) \delta(\bar{x}) .
$$

The leading order behaviour as $\tau \rightarrow 0$ is described by the solution of

$$
u_{0 \bar{\tau}}=\left(u_{0}^{3}\right)_{\bar{x} \bar{x}}, \quad u_{0}(\bar{x}, 0)=(\beta / \sqrt{2}) \delta(\bar{x}),
$$

and this is given by the Barenblatt-Pattle similarity solution

$$
u_{0}(\bar{x}, \bar{\tau})= \begin{cases}\frac{1}{\sqrt{12} \bar{\tau}^{\frac{1}{2}}}\left(c^{2} \bar{\tau}^{\frac{1}{2}}-\bar{x}^{2}\right)^{\frac{1}{2}}, & |\bar{x}|<c \bar{\tau}^{\frac{1}{4}} \\ 0 & |\bar{x}|>c \bar{\tau}^{\frac{1}{4}}\end{cases}
$$

where $c^{2}=2 \sqrt{6} \beta / \pi$. This solution, which is integrated twice to recover the original option value function, has compact support and indicates, in effect, that for short times near expiry, the option value is equal to the payoff (discounting takes effect at higher order) except in the sharply-defined small region $|S-K|<$ $K \lambda c\left(\sigma^{2}(T-t)\right)^{\frac{1}{4}}$; in the linear diffusion case, by contrast, the region where the option value is non-trivial merges smoothly into the outer region.

Still following [13], it is possible to push the analysis a little further by considering the transitions near $\bar{x}= \pm c \bar{\tau}^{\frac{1}{4}}$, via which the non-zero part of the solution above joins onto the zero parts. The Barenblatt-Pattle solution has infinite gradient at $\bar{x}= \pm c \bar{\tau}^{\frac{1}{4}}$ and vanishes for $|x|>c \bar{\tau}^{\frac{1}{4}}$, but the original differential equation for $u$ contains a linear term and its solution is strictly 
positive for positive initial data. The discrepancy is resolved as in a similar problem from a model of semiconductor fabrication [14] by introducing a further inner layer near (say) $\bar{x}=\bar{s}(\bar{\tau})=c \bar{\tau}^{\frac{1}{4}}$ in which $\bar{x}=\bar{s}(\bar{\tau})+\epsilon X, u=\epsilon^{\frac{1}{2}} U$, to find that

$$
\epsilon^{\frac{1}{2}} U_{\bar{\tau}}-\dot{s} U_{X}=\left(U^{3}+(\sqrt{2} / \beta) U^{2}+\frac{1}{2} U\right)_{X X}
$$

with $U \rightarrow 0$ as $X \rightarrow+\infty$ and matching with the Barenblatt-Pattle solution as $X \rightarrow-\infty$. To leading order the solution is the travelling wave $U(X, \bar{\tau})=U_{0}(X)$ where

$$
\dot{s} \frac{\mathrm{d} U_{0}}{\mathrm{~d} X}+\frac{\mathrm{d}^{2}}{\mathrm{~d} X^{2}}\left(U_{0}^{3}+(\sqrt{2} / \beta) U_{0}^{2}+\frac{1}{2} U_{0}\right)=0,
$$

so that integrating once and using the condition at $X=\infty$ we have

$$
\dot{s} U_{0}+\frac{\mathrm{d}}{\mathrm{d} X}\left(U_{0}^{3}+(\sqrt{2} / \beta) U_{0}^{2}+\frac{1}{2} U_{0}\right)=0 .
$$

A second integration shows that

$$
\frac{3}{2} U_{0}^{2}+\frac{2 \sqrt{2}}{\beta} U_{0}+\frac{1}{2} \log U_{0}=-\dot{s}\left(X-X_{0}\right),
$$

where $X_{0}$ is a centering constant which can only be determined at higher order. We therefore have exponential decay in the solution as $X \rightarrow+\infty$, while matching as $X \rightarrow-\infty$ shows that

$$
U_{0} \sim\left(-\frac{2}{3} \dot{s} X\right)^{\frac{1}{2}}
$$

which is indeed consistent with the expansion of the Barenblatt-Pattle solution near $x=s(\bar{\tau})=c \bar{\tau}^{\frac{1}{4}}$, and this completes the asymptotic analysis of this problem.

\section{Fast mean-reverting volatility}

Our final example of the applicability of boundary-layer techniques is in the analysis of fast-mean-reverting stochastic volatility models [15]. In these models the volatility itself is assumed to follow a stochastic process while the asset price is assumed to follow the lognormal process as before. That is, we assume that

$$
\frac{\mathrm{d} S_{t}}{S_{t}}=\mu \mathrm{d} t+\sigma_{t} \mathrm{~d} W_{t}, \quad d \sigma_{t}=M_{t} \mathrm{~d} t+\Sigma_{t} \mathrm{~d} \widetilde{W}_{t}
$$

where $M_{t}$ and $\Sigma$ are the drift and volatility of the volatility and the instantaneous correlation between the Brownian motions $W_{t}$ and $\widetilde{W}_{t}$ is denoted by $\rho$. A commonly used example is the Heston model [16]. This is

$$
d\left(\sigma_{t}^{2}\right)=-\kappa\left(\sigma_{t}^{2}-\bar{\sigma}_{\infty}^{2}\right) \mathrm{d} t+\theta \sigma_{t} \mathrm{~d} \widetilde{W}_{t}
$$


for constant $\kappa, \theta$ and $\bar{\sigma}_{\infty}$; it will be noted that the volatility exhibits meanreversion to the long-term level $\bar{\sigma}_{\infty}$. (Although the model is written in terms of the instantaneous variance $\sigma_{t}^{2}$, it can be transformed into one for $\sigma_{t}$ via the Itô formula.)

When using such a model, perfect hedging is no longer possible and there is no unique pricing measure. Instead, a standard analysis shows that there is a market price of volatility risk, denoted by $\lambda$ (which may be a function of any of $S, \sigma$ and $t$ ) and pricing is done with respect to the measure associated with the processes

$$
\frac{\mathrm{d} S_{t}}{S_{t}}=r \mathrm{~d} t+\sigma_{t} \mathrm{~d} W_{t}, \quad \mathrm{~d} \sigma_{t}=\left(M_{t}-\lambda \Sigma\right) \mathrm{d} t+\Sigma_{t} \mathrm{~d} \widetilde{W}_{t}
$$

In effect, the market price of volatility risk represents the extra return required by the market for taking on this (untradable) risk. A standard analysis then shows that the price $V(S, \sigma, t)$, which now depends on the instantaneous volatility $\sigma$ as well as the spot price and time, satisfies the backward parabolic equation $\frac{\partial V}{\partial t}+\frac{1}{2} \sigma^{2} S^{2} \frac{\partial^{2} V}{\partial S^{2}}+\rho S \sigma \Sigma \frac{\partial^{2} V}{\partial S \partial \sigma}+\frac{1}{2} \Sigma^{2} \frac{\partial^{2} V}{\partial \sigma^{2}}+r S \frac{\partial V}{\partial S}+(M-\lambda \Sigma) \frac{\partial V}{\partial \sigma}-r V=0$, which evidently contains the Black-Scholes equation as a special case.

We assume for simplicity (as is reasonable in practice) that the drift and volatility of the volatility are functions only of $\sigma$, not of $S$ or $t$. Let us consider the commonly occurring situation in which the volatility process is fast meanreverting, in which the timescale for mean-reversion is much shorter than that for the evolution of the asset price, their ratio $\epsilon$ being small. ${ }^{3}$ (In the Heston model above, this ratio is $\bar{\sigma}_{\infty}^{2} / \kappa$.) Then $M_{t}$ and $\Sigma_{t}$ are written

$$
M_{t}=\frac{m_{t}}{\epsilon}, \quad \Sigma_{t}=\frac{\varsigma_{t}}{\epsilon^{1 / 2}}
$$

the relative sizes of these coefficients are chosen so that $\sigma_{t}$ has a nontrivial invariant distribution

$$
\lim _{t \rightarrow \infty} p\left(\sigma_{t}, t \mid \sigma_{0}, 0\right)
$$

where $p\left(\sigma_{t}, t \mid \sigma_{0}, 0\right)$ is the transition density function for $\sigma_{t}$ starting from $\sigma_{0}$ at time zero, which satisfies the forward Kolmogorov equation

$$
\frac{\partial p}{\partial t}=\frac{\partial}{\partial \sigma}\left(\frac{1}{2} \Sigma^{2} \frac{\partial p}{\partial \sigma}\right)-\frac{\partial}{\partial \sigma}(M p) .
$$

We denote this time-independent invariant distribution by $p_{\infty}(\sigma)$.

With these assumptions the pricing equation becomes

$$
\frac{\partial V}{\partial t}+\frac{1}{2} \sigma^{2} S^{2} \frac{\partial^{2} V}{\partial S^{2}}+\frac{\rho \varsigma}{\epsilon^{\frac{1}{2}}} \sigma S \frac{\partial^{2} V}{\partial S \partial \sigma}+\frac{1}{2} \frac{\varsigma^{2}}{\epsilon} \frac{\partial^{2} V}{\partial \sigma^{2}}+r S \frac{\partial V}{\partial S}+\left(\frac{m}{\epsilon}-\frac{\lambda \varsigma}{\epsilon^{\frac{1}{2}}}\right) \frac{\partial V}{\partial \sigma}-r V=0 .
$$

\footnotetext{
${ }^{3}$ We use $\epsilon$ rather than $\epsilon^{2}$ (which would have been consistent with the usage of Section 3 ) in conformity with existing literature, especially [15, 17]. Note also that, for the same reason, we do not make the pricing equation dimensionless. In this problem, there is little advantage to be gained by so doing.
} 
It is possible to construct asymptotic approximations to the solutions of this equation, valid as $\epsilon \rightarrow 0$, and this approach was pioneered by Fouque, Papanicolaou \& Sircar; it is summarised in their book [15]. They construct approximations correct to $O\left(\epsilon^{\frac{1}{2}}\right)$ which are valid everywhere except in a boundary layer near expiry. In later work, Rasmussen \& Wilmott [18] extended the outer expansion to $O(\epsilon)$. However, the solution to this order contains an arbitrary function which can only be determined by a boundary layer analysis which they do not carry out, and this gap is filled in the current paper. Our analysis parallels that of [17] who analysed products whose payoff depends on some measure of the realised volatility and who also analysed the boundary layer, although they did not construct as many terms as are needed for the accuracy we now achieve. In related work, $[19,20]$ consider the question of convergence of the asymptotic series to the exact solution, and derive formulas similar to ours, although they do not exploit the boundary-layer structure. The work in [20], in particular, was carried out simultaneously with ours although independently of it, and derives similar formulas in a rather different setting. It is a pleasure to acknowledge helpful comments from one of the authors of this paper.

\subsection{The outer expansion}

We begin with the outer expansion. We write the fast mean-reverting pricing equation in the form

$$
\left(\frac{1}{\epsilon} \mathcal{L}_{0}+\frac{1}{\epsilon^{1 / 2}} \mathcal{L}_{\frac{1}{2}}+\mathcal{L}_{1}\right) V=0
$$

where

$$
\begin{gathered}
\mathcal{L}_{0}=\frac{1}{2} \varsigma^{2} \frac{\partial^{2}}{\partial \sigma^{2}}+m \frac{\partial}{\partial \sigma}, \\
\mathcal{L}_{\frac{1}{2}}=\rho \varsigma \sigma S \frac{\partial^{2}}{\partial S \partial \sigma}-\lambda \varsigma \frac{\partial}{\partial \sigma}, \\
\mathcal{L}_{1}=\frac{\partial}{\partial t}+\frac{1}{2} \sigma^{2} S^{2} \frac{\partial^{2}}{\partial S^{2}}+r S \frac{\partial}{\partial S}-r ;
\end{gathered}
$$

note that $\mathcal{L}_{1}$ is the Black-Scholes operator with volatility $\sigma$. Because $\mathcal{L}_{0}$ is the generator of the backward Kolmogorov equation for $\sigma_{t}$, its adjoint $\mathcal{L}_{0}^{*}$ is the generator of the forward equation and so $p_{\infty}(\sigma)$ satisfies

$$
\mathcal{L}_{0}^{*} p_{\infty}=\frac{\partial^{2}}{\partial \sigma^{2}}\left(\frac{1}{2} \varsigma^{2} p_{\infty}\right)-\frac{\partial}{\partial \sigma}\left(m p_{\infty}\right)=0 .
$$

Assuming that $\varsigma^{2}, m$ are such that $p_{\infty}$ exists, it is then proportional to

$$
e^{-2 \int^{\sigma} m(s) / \varsigma^{2}(s) \mathrm{d} s} / \varsigma^{2}(\sigma)
$$

The standard integration-by-parts identity

$$
\left\langle\mathcal{L}_{0} u, v\right\rangle=-\left\langle u, \mathcal{L}_{0}^{*} v\right\rangle
$$


for suitable functions $u$ and $v$, where $\langle\cdot, \cdot\rangle$ is the usual inner product over $0<\sigma<\infty$, will prove useful.

We now expand

$V(S, \sigma, t) \sim V_{0}(S, \sigma, t)+\epsilon^{\frac{1}{2}} V_{\frac{1}{2}}(S, \sigma, t)+\epsilon V_{1}(S, \sigma, t)+\epsilon^{\frac{3}{2}} V_{\frac{3}{2}}(S, \sigma, t)+\epsilon^{2} V_{2}(S, \sigma, t)+\cdots$

and substitute into the pricing equation. Equating coefficients of powers of $\epsilon$, we obtain in increasing order of the power the equations

$$
\begin{gathered}
\mathcal{L}_{0} V_{0}=0, \\
\mathcal{L}_{0} V_{\frac{1}{2}}+\mathcal{L}_{\frac{1}{2}} V_{0}=0, \\
\mathcal{L}_{0} V_{1}+\mathcal{L}_{\frac{1}{2}} V_{\frac{1}{2}}+\mathcal{L}_{1} V_{0}=0, \\
\mathcal{L}_{0} V_{\frac{3}{2}}+\mathcal{L}_{\frac{1}{2}} V_{1}+\mathcal{L}_{1} V_{\frac{1}{2}}=0, \\
\mathcal{L}_{0} V_{2}+\mathcal{L}_{\frac{1}{2}} V_{\frac{3}{2}}+\mathcal{L}_{1} V_{1}=0 .
\end{gathered}
$$

At lowest order, we have $\mathcal{L}_{0} V_{0}=0$ and so $V_{0}$ is a function $\mathcal{V}_{0}(S, t)$ of $S$ and $t$ alone, since $\mathcal{L}_{0}$ consists only of $\sigma$-derivatives (the particular solutions that depend on $\sigma$ are ruled out by the conditions at large and/or small $S$ ). However, $\mathcal{V}_{0}$ is as yet undetermined and this is the principal feature of the analysis. In approximating, we have replaced the non-degenerate stochastic volatility differential operator by the degenerate operator $\mathcal{L}_{0}$ which has no $S$ or $t$ derivatives. ${ }^{4}$ In consequence, the solution contains eigenfunctions (of which $\mathcal{V}_{0}(S, t)$ is one) which can only be determined by application of the Fredholm Alternative at higher order.

As $V_{0}$ is a function of $(S, t)$ alone, we have $\mathcal{L}_{\frac{1}{2}} V_{0}=0$ and so $V_{\frac{1}{2}}$ is also a function, also as yet unknown, of $(S, t)$ alone, $\mathcal{V}_{\frac{1}{2}}(S, t)$. Thus $\mathcal{L}_{\frac{1}{2}} V_{\frac{1}{2}}=0$ and so we have

$$
\mathcal{L}_{0} V_{1}=-\mathcal{L}_{1} V_{0} .
$$

Because the operator $\mathcal{L}_{0}$ is degenerate, by the Fredholm Alternative this equation only has solutions if the right-hand side is orthogonal to the relevant eigenfunction of $\mathcal{L}_{0}^{*}$, namely $p_{\infty}$. Thus, $\left\langle\mathcal{L}_{1} V_{0}, p_{\infty}\right\rangle=0$; carrying out the integration, bearing in mind that the only $\sigma$-dependence is in the volatility coefficient $\sigma^{2}$, we find that

$$
\overline{\mathcal{L}_{1}} V_{0}=\left\langle\mathcal{L}_{1} V_{0}, p_{\infty}\right\rangle=\frac{\partial \mathcal{V}_{0}}{\partial t}+\frac{1}{2} \overline{\sigma^{2}} S^{2} \frac{\partial^{2} \mathcal{V}_{0}}{\partial S^{2}}+r S \frac{\partial \mathcal{V}_{0}}{\partial S}-r \mathcal{V}_{0}=0,
$$

where $\overline{\sigma^{2}}=\left\langle\sigma^{2}, p_{\infty}\right\rangle=\int_{0}^{\infty} \sigma^{2} p_{\infty}(\sigma) \mathrm{d} \sigma$ (the notation $\overline{\sigma^{2}}$ thus represents the average of $\sigma^{2}$ with respect to the invariant distribution $\left.p_{\infty}\right)$. This is the BlackScholes equation with averaged volatility $\left(\overline{\sigma^{2}}\right)^{1 / 2}$. The solution can be calculated by standard Black-Scholes techniques once a suitable final value is given. As we show below, this value is the payoff $P(S)$, and so $V_{0}(S, \sigma, t)=\mathcal{V}_{0}(S, t)$

\footnotetext{
${ }^{4} \mathrm{~A}$ similar situation occurs when, in a classical 'lubrication theory' analysis in a long thin domain, an elliptic operator is replaced by a parabolic one [21].
} 
is the Black-Scholes value of the option with constant volatility equal to the square root of the stationary mean of the variance. This is intuitively reasonable in view of the fact (see [5]) that if the volatility in a Black-Scholes equation is a given deterministic function $\sigma(t)$ of time, the option value can be calculated by replacing $\sigma$ in the relevant constant-volatility formula by

$$
\left(\frac{1}{T-t} \int_{t}^{T} \sigma^{2}(s) \mathrm{d} s\right)^{\frac{1}{2}}
$$

so that we have, in effect, an application of the law of large numbers.

We now calculate $V_{1}$. As $\overline{\mathcal{L}_{1}} V_{0}=0$, we have by subtraction

$$
\mathcal{L}_{1} V_{0}=\mathcal{L}_{1} V_{0}-\overline{\mathcal{L}_{1}} V_{0}=\frac{1}{2}\left(\sigma^{2}-\overline{\sigma^{2}}\right) S^{2} \frac{\partial^{2} \mathcal{V}_{0}}{\partial S^{2}}
$$

thereby replacing the majority of the source terms in $\mathcal{L}_{0} V_{1}$, leaving

$$
\mathcal{L}_{0} V_{1}=\frac{1}{2}\left(\overline{\sigma^{2}}-\sigma^{2}\right) S^{2} \frac{\partial^{2} \mathcal{V}_{0}}{\partial S^{2}} .
$$

The solution of this equation has the form

$$
V_{1}(S, \sigma, t)=g_{1}(\sigma) S^{2} \frac{\partial^{2} \mathcal{V}_{0}}{\partial S^{2}}+\mathcal{V}_{1}(S, t)
$$

where $\mathcal{V}_{1}(S, t)$ is another eigenfunction of $\mathcal{L}_{0}$ which can only be determined by a solvability condition at the next order; $g_{1}(\sigma)$ satisfies

$$
\frac{1}{2} \varsigma^{2}(\sigma) \frac{\mathrm{d}^{2} g_{1}}{\mathrm{~d} \sigma^{2}}+m(\sigma) \frac{\mathrm{d} g_{1}}{\mathrm{~d} \sigma}=\frac{1}{2}\left(\overline{\sigma^{2}}-\sigma^{2}\right)
$$

and can be written in integral form (one of the "complementary solutions" is a constant and can be absorbed into $\mathcal{V}_{1}(S, t)$, and the other is unbounded at infinity).

Although we have calculated $V_{0}$, at this stage $V_{\frac{1}{2}}$ and $V_{1}$ are still undetermined to within an eigenfunction, and furthermore $V_{1}$ depends explicitly on $\sigma$, which is not consistent with a $\sigma$-independent payoff $P(S)$. We deal with the former difficulty by going to higher order in the expansion, and the latter by a boundary layer analysis.

Before proceeding further, we outline the pattern followed by successive iterations of the solution procedure. We first solve equation $n$, namely $\mathcal{L}_{0} V_{n}=$ $-\mathcal{L}_{\frac{1}{2}} V_{n-\frac{1}{2}}-\mathcal{L}_{1} V_{n-1}$ for $V_{n}(S, \sigma, t)$ (the right-hand side is assumed known from earlier stages), finding a particular solution $v_{n}(S, \sigma, t)$ and an eigenfunction $\mathcal{V}_{n}(S, t)$ (for the case $n=0$ dealt with above, the former is zero and the latter is $\left.\mathcal{V}_{0}(S, t)\right)$. We repeat this process for $V_{n+\frac{1}{2}}$, obtaining a further particular solution $v_{n+\frac{1}{2}}$ and a further eigensolution $\mathcal{V}_{n+\frac{1}{2}}$ (note that the eigensolution $\mathcal{V}_{n}$ is annihilated by $\left.\mathcal{L}_{0}\right)$. Finally we substitute the functions just found into the right-hand side of the equation for $V_{n+1}$, namely $\mathcal{L}_{0} V_{n+1}=-\mathcal{L}_{\frac{1}{2}} V_{n+\frac{1}{2}}-\mathcal{L}_{1} V_{n}$; 
again the eigenfunction $\mathcal{V}_{n+\frac{1}{2}}$ is annihilated, so the right-hand side is known in terms of the particular solutions just found. We can now apply the solvability condition $\left\langle\mathcal{L}_{0} V_{n+1}, p_{\infty}\right\rangle=0$ for existence of a solution to obtain an equation from which the eigenfunction $\mathcal{V}_{n}(S, t)$ can, in principle and given appropriate terminal conditions, be found; this equation is

$$
\overline{\mathcal{L}_{1}} \mathcal{V}_{n}=-\overline{\mathcal{L}_{\frac{1}{2}} v_{n+\frac{1}{2}}}-\overline{\mathcal{L}_{1} v_{n}}
$$

Having dealt with $n=0$ above, we now apply this procedure to the case $n=$ $\frac{1}{2}$. We have already found $V_{\frac{1}{2}}(S, \sigma, t)$ and $V_{1}(S, \sigma, t)$ up to eigenfunctions, so we need only apply the solvability condition to the equation $\mathcal{L}_{0} V_{\frac{3}{2}}=-\mathcal{L}_{\frac{1}{2}} V_{1}-\mathcal{L}_{1} V_{\frac{1}{2}}$, noting that $\mathcal{L}_{\frac{1}{2}} \mathcal{V}_{1}=0$. This gives

$$
\overline{\mathcal{L}_{1}} V_{\frac{1}{2}}=-\overline{\rho \varsigma \sigma g_{1}^{\prime}} S \frac{\partial}{\partial S}\left(S^{2} \frac{\partial^{2} \mathcal{V}_{0}}{\partial S^{2}}\right)+\overline{\lambda \varsigma g_{1}^{\prime}} S^{2} \frac{\partial^{2} \mathcal{V}_{0}}{\partial S^{2}}
$$

where $g_{1}^{\prime}=\mathrm{d} g_{1} / \mathrm{d} \sigma$. Using a manoeuvre similar to that employed for the heat equation in Section 3, and assuming pro tem that the correct final condition (from matching) is $V_{\frac{1}{2}}(S, T)=0$, we find

$$
V_{\frac{1}{2}}(S, \sigma, t)=(T-t)\left(A_{\frac{1}{2}, 1} S^{2} \frac{\partial^{2} \mathcal{V}_{0}}{\partial S^{2}}+A_{\frac{1}{2}, 2} S \frac{\partial}{\partial S}\left(S^{2} \frac{\partial^{2} \mathcal{V}_{0}}{\partial S^{2}}\right)\right)
$$

where $\mathcal{V}_{0}$ is already known and $A_{\frac{1}{2}, 1}=\overline{\lambda \varsigma g_{1}^{\prime}}, A_{\frac{1}{2}, 2}=-\overline{\rho \varsigma \sigma g_{1}^{\prime}}$ (as noted earlier, $V_{\frac{1}{2}}$ is independent of $\sigma$ ). This result, and elaborations thereof, is an important practical consequence of the method since, as described in [15], it allows calibration of the three constants $\overline{\sigma^{2}}, \overline{\rho \varsigma \sigma g_{1}^{\prime}}$ and $\overline{\lambda \varsigma g_{1}^{\prime}}$ to market prices of options (as represented by an implied volatility surface) in a simple manner: the key point is that only these directly deducable constants are needed, rather than the unobservable functions $M(\sigma, t)$ and $\Sigma(\sigma, t)$.

We need to carry out one more iteration. For ease of notation, we set

$$
\mathcal{D}=S \frac{\partial}{\partial S}
$$

equivalent to using a logarithmic price variable as in [20]. We first calculate

$$
\begin{gathered}
\mathcal{L}_{0} V_{\frac{3}{2}}=\left[\varsigma g_{1}^{\prime}(\lambda-\rho \sigma \mathcal{D})+\frac{1}{2}\left(\overline{\sigma^{2}}-\sigma^{2}\right)(T-t)\left(A_{\frac{1}{2}, 1}+A_{\frac{1}{2}, 2} \mathcal{D}\right)\left(\mathcal{D}^{2}-\mathcal{D}\right)\right. \\
\left.-A_{\frac{1}{2}, 1}-A_{\frac{1}{2}, 2} \mathcal{D}\right]\left(\mathcal{D}^{2}-\mathcal{D}\right) \mathcal{V}_{0}
\end{gathered}
$$

From this, we find $V_{\frac{3}{2}}$ in the form

$$
V_{\frac{3}{2}}(S, \sigma, t)=\left[g_{2}(\sigma)+g_{3}(\sigma) \mathcal{D}+g_{1}(\sigma)(T-t)\left(A_{\frac{1}{2}, 1}+A_{\frac{1}{2}, 2} \mathcal{D}\right)\left(\mathcal{D}^{2}-\mathcal{D}\right)\right]\left(\mathcal{D}^{2}-\mathcal{D}\right) \mathcal{V}_{0}
$$

where $g_{2}(\sigma)$ and $g_{3}(\sigma)$ satisfy the same equation as $g_{1}(\sigma)$ but with the relevant inhomogeneous terms taken from the equation for $V_{\frac{3}{2}}$; that is, for $g_{2}(\sigma)$ the 
inhomogeneous term is $\lambda \varsigma g_{1}^{\prime}(\sigma)-A_{\frac{1}{2}, 1}$, and for $g_{2}(\sigma)$ it is $-\rho \sigma \varsigma g_{1}^{\prime}(\sigma)-A_{\frac{1}{2}, 2}$ (recall that $\rho, \lambda$ and $\varsigma$ may depend on $\sigma$ ). Then, the solvability condition for $V_{2}$, applied to the equation $\mathcal{L}_{0} V_{2}=-\mathcal{L}_{\frac{1}{2}} V_{\frac{3}{2}}-\mathcal{L}_{1} V_{1}$, gives, after some calculation,

$$
\begin{aligned}
\overline{\mathcal{L}_{1}} \mathcal{V}_{1}=[ & \left(A_{1,1}+A_{1,2} \mathcal{D}\right) \mathcal{D} \\
& \left.+(T-t)\left(A_{\frac{1}{2}, 1}+A_{\frac{1}{2}, 2} \mathcal{D}\left(\mathcal{D}^{2}-\mathcal{D}\right)\right)\left(A_{\frac{1}{2}, 1}+A_{\frac{1}{2}, 2} \mathcal{D}\right)\right]\left(\mathcal{D}^{2}-\mathcal{D}\right) \mathcal{V}_{0},
\end{aligned}
$$

where the new constant coefficients are

$$
A_{1,1}=\overline{\varsigma g_{2}^{\prime}(\sigma)(\lambda-\rho \sigma)}, \quad A_{1,2}=\overline{\varsigma g_{3}^{\prime}(\sigma)(\lambda-\rho \sigma)} .
$$

The relevant particular solution with zero payoff condition is

$$
\begin{aligned}
-[(T-t)( & \left.A_{1,1}+A_{1,2} \mathcal{D}\right) \mathcal{D} \\
& \left.+\frac{1}{2}(T-t)^{2}\left(A_{\frac{1}{2}, 1}+A_{\frac{1}{2}, 2} \mathcal{D}\left(\mathcal{D}^{2}-\mathcal{D}\right)\right)\left(A_{\frac{1}{2}, 1}+A_{\frac{1}{2}, 2} \mathcal{D}\right)\right]\left(\mathcal{D}^{2}-\mathcal{D}\right) \mathcal{V}_{0}
\end{aligned}
$$

however, we leave open the possibility of adding a further solution $\mathcal{V}_{1}^{\prime}(S, t)$ if the payoff, determined by matching into the boundary layer, dictates that we should do so. Likewise, the $\sigma$-dependence of the solution can only be resolved by matching.

\subsection{Boundary layer analysis}

We introduce a boundary layer in $t$ near $t=T$, of size $O(\epsilon)$, defining the inner time variable $\tau$ via

$$
t=T+\epsilon \tau, \quad \tau<0,
$$

so that the pricing equation for $\widetilde{V}(S, \sigma, \tau)=V(S, \sigma, t)$ is

$\frac{1}{\epsilon} \frac{\partial V}{\partial \tau}+\frac{1}{2} \sigma^{2} S^{2} \frac{\partial^{2} V}{\partial S^{2}}+\frac{\rho \varsigma}{\epsilon^{\frac{1}{2}}} \sigma S \frac{\partial^{2} V}{\partial S \partial \sigma}+\frac{1}{2} \frac{\varsigma^{2}}{\epsilon} \frac{\partial^{2} V}{\partial \sigma^{2}}+r S \frac{\partial V}{\partial S}+\left(\frac{m}{\epsilon}-\frac{\lambda \varsigma}{\epsilon^{\frac{1}{2}}}\right) \frac{\partial V}{\partial \sigma}-r V=0$.

We write this as

$$
\left(\frac{1}{\epsilon} \widetilde{\mathcal{L}}_{0}+\frac{1}{\epsilon^{1 / 2}} \widetilde{\mathcal{L}}_{\frac{1}{2}}+\widetilde{\mathcal{L}}_{1}\right) \widetilde{V}=0
$$

where

$$
\begin{gathered}
\widetilde{\mathcal{L}}_{0}=\frac{\partial}{\partial \tau}+\mathcal{L}_{0}=\frac{\partial}{\partial \tau}+\frac{1}{2} \varsigma^{2} \frac{\partial^{2}}{\partial \sigma^{2}}+m \frac{\partial}{\partial \sigma}, \\
\widetilde{\mathcal{L}}_{\frac{1}{2}}=\mathcal{L}_{\frac{1}{2}}=\rho \varsigma \sigma S \frac{\partial^{2}}{\partial S \partial \sigma}-\lambda \varsigma \frac{\partial}{\partial \sigma}, \\
\widetilde{\mathcal{L}}_{1}=\frac{1}{2} \sigma^{2} S^{2} \frac{\partial^{2}}{\partial S^{2}}+r S \frac{\partial}{\partial S}-r,
\end{gathered}
$$


(note that $\widetilde{\mathcal{L}}_{0}$, unlike $\mathcal{L}_{0}$, contains the time derivative $\partial / \partial \tau$; this effectively removes the degeneracy that made the outer expansion so complicated) and we expand

$$
\widetilde{V} \sim \widetilde{V}_{0}+\epsilon^{1 / 2} \widetilde{V}_{\frac{1}{2}}+\epsilon \widetilde{V}_{1}+\cdots .
$$

At leading order we have the one-term inner solution which satisfies

$$
\widetilde{\mathcal{L}}_{0} \widetilde{V}_{0}=0, \quad \widetilde{V}_{0}(S, \sigma, 0)=P(S)
$$

the solution is

$$
\widetilde{V}_{0}(S, \sigma, \tau)=P(S)
$$

and it matches automatically with the one-term outer solution $V_{0}(S, t)$ as $t \rightarrow T$, $\tau \rightarrow-\infty$.

At the next order, we have

$$
\widetilde{\mathcal{L}}_{0} \widetilde{V}_{\frac{1}{2}}=-\mathcal{L}_{\frac{1}{2}} \widetilde{V}_{0}=0, \quad \widetilde{V}_{\frac{1}{2}}(S, T)=0 ;
$$

the solution is $\widetilde{V}_{\frac{1}{2}}=0$, and this is consistent with matching with the two-term outer expansion $V_{0}+\epsilon^{1 / 2} V_{\frac{1}{2}}$, since in inner variables the $T-t$ in $V_{\frac{1}{2}}$ means that this term only contributes $O\left(\epsilon^{3 / 2}\right)$ to the inner expansion of the outer solution. We now see the correctness of ignoring the possible added eigenfunction at this order in the outer expansion.

At the next order we have

$$
\widetilde{\mathcal{L}}_{0} \widetilde{V}_{1}=-\widetilde{\mathcal{L}}_{1} \widetilde{V}_{0}=-\widetilde{\mathcal{L}}_{1} P, \quad \widetilde{V}_{1}(S, \sigma, 0)=0,
$$

which, noting that $\partial P / \partial \tau=0$, can be written as

$$
\frac{\partial \widetilde{V}_{1}}{\partial \tau}+\mathcal{L}_{0} \widetilde{V}_{1}=\frac{1}{2}\left(\overline{\sigma^{2}}-\sigma^{2}\right) S^{2} \frac{\partial^{2} P}{\partial S^{2}}-\overline{\mathcal{L}}_{1} P, \quad \widetilde{V}_{1}(S, \sigma, 0)=0 .
$$

We now need the limiting behaviour of the solution of this equation as $\tau \rightarrow$ $-\infty$ in order to match with the three-term outer solution. A particular solution is

$$
\widetilde{V}_{1}^{\infty}=g_{1}(\sigma) S^{2} \frac{\partial^{2} P}{\partial S^{2}}-\tau \overline{\mathcal{L}}_{1} P+\widetilde{\mathcal{V}}_{1}(S)
$$

where $\widetilde{\mathcal{V}}_{1}(S)$ is arbitrary, and this is in fact the correct form for the asymptotic behaviour of $\widetilde{V}_{1}(S, \sigma, \tau)$ as $\tau \rightarrow-\infty$. To see this, we first note that

$$
\left\langle\widetilde{\mathcal{L}}_{0} \widetilde{V}_{1}, p_{\infty}\right\rangle=\left\langle\frac{\partial \widetilde{V}_{1}}{\partial \tau}+\mathcal{L}_{0} \widetilde{V}_{1}, p_{\infty}\right\rangle=\left\langle\frac{\partial \widetilde{V}_{1}}{\partial \tau}, p_{\infty}\right\rangle
$$

since $\left\langle\mathcal{L}_{0} \widetilde{V}_{1}, p_{\infty}\right\rangle=0$. Furthermore,

$$
\frac{\partial}{\partial \tau}\left\langle\widetilde{V}_{1}, p_{\infty}\right\rangle=\left\langle\frac{\partial \widetilde{V}_{1}}{\partial \tau}, p_{\infty}\right\rangle=-\left\langle\overline{\mathcal{L}}_{1} P, p_{\infty}\right\rangle=-\overline{\mathcal{L}}_{1} P
$$


and so, integrating and using $\widetilde{V}_{1}(S, \sigma, 0)=0$,

$$
\left\langle\widetilde{V}_{1}, p_{\infty}\right\rangle=-\tau \overline{\mathcal{L}}_{1} P .
$$

However, using the solution found for $\widetilde{V}_{1}$, we also have that

$$
\left\langle\widetilde{V}_{1}^{\infty}, p_{\infty}\right\rangle=-\tau \overline{\mathcal{L}}_{1} P+\overline{g_{1}(\sigma)} S^{2} \frac{\partial^{2} P}{\partial S^{2}}+\widetilde{\mathcal{V}}_{1}(S) .
$$

Comparing these two expressions for the inner product, we see that

$$
\widetilde{\mathcal{V}}_{1}(S)=-\overline{g_{1}(\sigma)} S^{2} \frac{\partial^{2} P}{\partial S^{2}} .
$$

Thus, as $\tau \rightarrow-\infty$,

$$
V_{1} \sim \widetilde{V}_{1}^{\infty}=\left(g_{1}(\sigma)-\overline{g_{1}(\sigma)}\right) S^{2} \frac{\partial^{2} P}{\partial S^{2}}-\tau \overline{\mathcal{L}}_{1} P
$$

since what is left after subtracting the particular solution, $\widetilde{V}_{1}-\widetilde{V}_{1}^{\infty}$, satisfies the homogeneous version of the parabolic equation, has initial data that vanishes at large and small $\sigma$, and therefore vanishes as $\tau \rightarrow-\infty$.

\subsection{Matching}

We can now complete the matching. From the outer expansion, written in inner variables, we have

$$
\begin{aligned}
V_{0}(S, \sigma, T+\epsilon \tau) & +\epsilon^{\frac{1}{2}} V_{\frac{1}{2}}(S, \sigma, T+\epsilon \tau)+\epsilon V_{1}(S, \sigma, T+\epsilon \tau) \\
& \sim V_{0}(S, \sigma, T)+\epsilon^{\frac{1}{2}} V_{\frac{1}{2}}(S, \sigma, T)+\epsilon\left(\tau \frac{\partial V_{0}}{\partial t}(S, \sigma, T)+V_{1}(S, \sigma, T)\right) \\
& =\mathcal{V}_{0}(S, T)+\epsilon\left(\tau \frac{\partial \mathcal{V}_{0}}{\partial t}(S, T)+g_{1}(\sigma) S^{2} \mathcal{V}_{0 S S}+\mathcal{V}_{1}^{\prime}(S, T)\right) \\
& =P(S)+\epsilon\left(-\tau \overline{\mathcal{L}_{1}} P(S)+g_{1}(\sigma) S^{2} \mathcal{V}_{0 S S}+\mathcal{V}_{1}^{\prime}(S, T)\right) ;
\end{aligned}
$$

because, from one-term matching, $\mathcal{V}_{0}(S, T)=P(S)$ and $\overline{\mathcal{L}_{1}} \mathcal{V}_{0}=0$, we have $\partial \mathcal{V}_{0} / \partial t=-\overline{\mathcal{L}_{1}} P$, a result used in deriving the last line of this equation. This is the three-term outer expansion, in inner variables; note that the particular solutions in $V_{\frac{1}{2}}$ and $V_{1}$ that are multiplied by $T-t$ do not contribute to it.

As demonstrated in Section 5.2, the large- $\tau$ behaviour of the three-term inner expansion is

$$
P(S, I)+\epsilon\left(-\tau \overline{\mathcal{L}_{1}} P+\left(g_{1}(\sigma)-\overline{g_{1}(\sigma)}\right) S^{2} \frac{\partial^{2} P}{\partial S^{2}}\right) .
$$

Matching these two expressions, the missing final condition for $\mathcal{V}_{1}^{\prime}(S, t)$ is

$$
\mathcal{V}_{1}^{\prime}(S, T)=-\overline{g_{1}(\sigma)} S^{2} \frac{\partial^{2} P}{\partial S^{2}} .
$$


Hence, as $S^{2} \mathcal{V}_{0 S S}$ is itself a solution of $\overline{\mathcal{L}_{1}} V=0$, we have

$$
\mathcal{V}_{1}^{\prime}(S, t)=-\overline{g_{1}(\sigma)} S^{2} \frac{\partial^{2} \mathcal{V}_{0}}{\partial S^{2}}
$$

and the complete outer expansion to three terms is

$$
\begin{aligned}
& V(S, \sigma, t) \sim \mathcal{V}_{0}(S, t)+\epsilon^{\frac{1}{2}}(T-t)\left(A_{\frac{1}{2}, 1}+A_{\frac{1}{2}, 2} \mathcal{D}\right)\left(\mathcal{D}^{2}-\mathcal{D}\right) \mathcal{V}_{0} \\
& +\epsilon\left[g_{1}(\sigma)-\overline{g_{1}(\sigma)}-(T-t)\left(A_{1,1}+A_{1,2} \mathcal{D}\right) \mathcal{D}\right. \\
& \left.\quad-\frac{1}{2}(T-t)^{2}\left(A_{\frac{1}{2}, 1}+A_{\frac{1}{2}, 2} \mathcal{D}\left(\mathcal{D}^{2}-\mathcal{D}\right)\right)\left(A_{\frac{1}{2}, 1}+A_{\frac{1}{2}, 2} \mathcal{D}\right)\right]\left(\mathcal{D}^{2}-\mathcal{D}\right) \mathcal{V}_{0}
\end{aligned}
$$

again, we recall that $\mathcal{D}=S \partial / \partial S$.

This result can be used to refine the $O\left(\epsilon^{\frac{1}{2}}\right)$ calibration [15] to market data; it should be noted that at $O(\epsilon)$ this involves the determination not only of the two new constants $A_{1,1}$ and $A_{1,2}$ but also of the function $g_{1}(\sigma)-\overline{g_{1}(\sigma)}$. In practice, the contribution from the latter is likely to be relatively small as the average of $g_{1}(\sigma)$ is subtracted from $g_{1}(\sigma)$ itself. The calibration is described in detail in $[20]$.

The analysis just presented must be modified if the payoff has a gradient discontinuity (or a jump), as then $S^{2} \mathcal{V}_{0 S S}$ is large near expiry for certain values of $S$; an example would be a call option with large Gamma near the strike $K$. This case can be treated by invoking a further inner region, in which $S=$ $K\left(1+\epsilon^{\frac{1}{2}} x\right), t=T+\epsilon \tau$, in which the leading order equation to be solved for $v(x, \sigma, \tau)=V(S, \sigma, t)$ is

$$
\frac{\partial v}{\partial \tau}+\frac{1}{2} \sigma^{2} \frac{\partial^{2} v}{\partial x^{2}}+\frac{1}{2} \varsigma^{2} \frac{\partial^{2} v}{\partial \sigma^{2}}+m \frac{\partial v}{\partial \sigma}=0
$$

with $v(x, \sigma, 0)=\max (x, 0)$. Unfortunately the coupling via the coefficient of $\partial^{2} v / \partial x^{2}$ means that explicit progress is unlikely for this problem, although its very short-time behaviour will be as in Section 3 .

\section{Discussion}

We have discussed a number of applications of the method of matched asymptotic expansions in finance, focusing on European vanilla options in several models. It is clear that the method can be applied in a much wider variety of situations, and although few of these opportunities have yet been exploited, there is corresponding work on American put options close to expiry where both conventional asymptotics and ray methods have been applied (see the recent paper [22] and references therein) and on discretely sampled barrier options [23]. But perhaps the most intriguing possibility is that of translating the techniques of matched asymptotic expansions so that they apply directly to the stochastic 
processes, rather than going via the partial differential equation for the probability density. For example, in Section 5, we introduced the process

$$
\mathrm{d} \sigma_{t}=-\frac{m}{\epsilon} \mathrm{d} t+\frac{\varsigma}{\epsilon^{\frac{1}{2}}} \mathrm{~d} W_{t} .
$$

for a fast mean-reverting volatility. Given the starting value $\sigma_{0}$ of $\sigma_{t}$, it is intuitively clear that over a timescale of $O(\epsilon)$ the exponential decay associated with the mean reversion acts to 'erase' the initial condition and that for longer timescales, say $t=O(1)$, all that we can say at time $t$ is that $\sigma_{t}$ is sampled from its stationary distribution $p_{\infty}(\cdot)$; the 'memory' of the initial condition is then $O\left(e^{-m t / \epsilon}\right)$, i.e. exponentially small. Similarly, the values of $\sigma_{t}$ at two times $t_{1}$ and $t_{2}$ separated by $O(1)$ are effectively independent samples from $p_{\infty}$ even though they are linked by a sample path.

To make this concept more precise, we first need to extend the traditional definition of an asymptotic expansion, which we do in conformity with the standard weak (in distribution) and strong (pathwise) convergence for stochastic processes. We propose the definition that a stochastic process $X_{t}^{\epsilon}$ depending on a parameter $\epsilon$ has the expansion

$$
X_{t}^{\epsilon} \stackrel{P}{\sim} X_{t}^{(0)}+\epsilon X_{t}^{(1)}+\epsilon^{2} X_{t}^{(2)}+\cdots \quad \text { as } \epsilon \rightarrow 0
$$

over a given time interval if, for each fixed $t$ in this interval, one can find processes $X_{t}^{(0)}, X_{t}^{(1)}, \ldots$ such that, for each $N$, the error

$$
\frac{1}{\epsilon^{N}}\left(X_{t}^{\epsilon}-\sum_{j=1}^{N} \epsilon^{j} X_{t}^{(j)}\right)
$$

converges in probability to zero as $\epsilon \rightarrow 0$. This is a pathwise asymptotic expansion in the sense that the sample paths of the expansion converge to those of the target process $X_{t}^{\epsilon}$, and it corresponds to a conventional regular expansion. Likewise we propose that

$$
X_{t}^{\epsilon} \stackrel{D}{\sim} X_{t}^{(0)}+\epsilon X_{t}^{(1)}+\epsilon^{2} X_{t}^{(2)}+\cdots \quad \text { as } \epsilon \rightarrow 0
$$

if the same error converges to zero in distribution as $\epsilon \rightarrow 0$, interpreted as

$$
\left|F_{S_{t}^{(N)}}-F_{X_{t}^{\epsilon}}\right| / \epsilon^{N} \rightarrow 0 \quad \text { as } \epsilon \rightarrow 0,
$$

where $S_{t}^{(N)}$ is the sum of the first $N$ terms in the expansion and $F$ is the cumulative density function. We term this type of expansion distributional.

By way of example, consider the slowly mean-reverting Ornstein-Uhlenbeck $(\mathrm{O}-\mathrm{U})$ process

$$
\mathrm{d} X_{t}=-\epsilon X_{t} \mathrm{~d} t+\mathrm{d} W_{t},
$$

with the starting value $X_{0}=x_{0}$ given. Posing a regular (pathwise) expansion as above, and equating coefficients of powers of $\epsilon$ in the usual way, we find the set of equations

$$
\mathrm{d} X_{t}^{(0)}=\mathrm{d} W_{t}, \quad X_{0}^{(0)}=x_{0},
$$




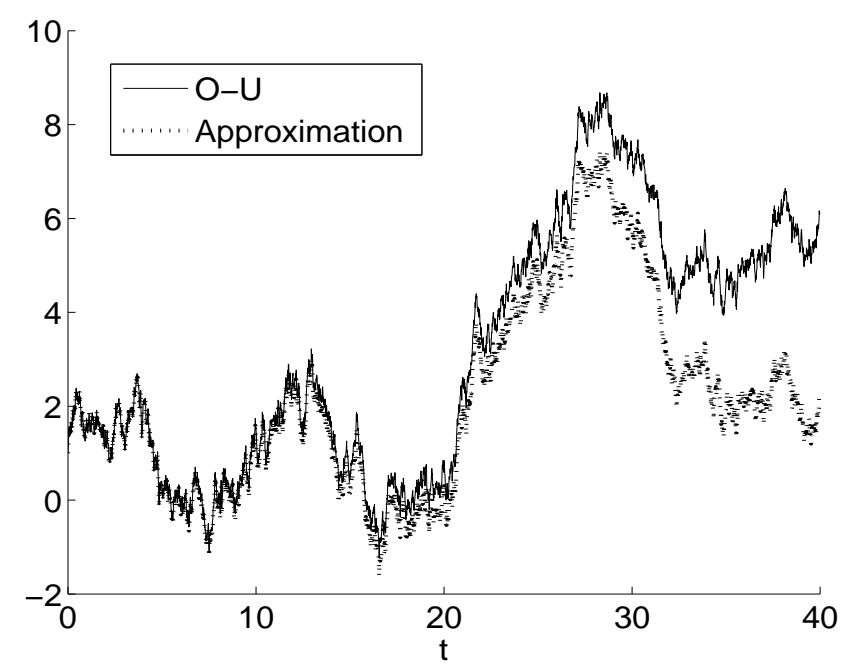

Figure 4: Numerical simulation, by explicit forward scheme with timestep 0.01, of the $\mathrm{O}-\mathrm{U}$ process $\mathrm{d} X_{t}=-\epsilon X_{t} \mathrm{~d} t+\mathrm{d} W_{t}, \epsilon=0.05$, and of the two-term asymptotic approximation. Divergence clearly becomes significant when $\epsilon t=$ $O(1)$.

$$
\mathrm{d} X_{t}^{(1)}=-X_{t}^{(0)} \mathrm{d} t, \quad X_{0}^{(1)}=0,
$$

and so on; their solutions are

$$
X_{t}^{(0)}=x_{0}+W_{t}, \quad X_{t}^{(1)}=-x_{0} t-\int_{0}^{t} W_{s} \mathrm{~d} s
$$

and these are in accordance with the exact solution, obtained with an integrating factor:

$$
\begin{aligned}
X_{t}^{\epsilon} & =x_{0} e^{-\epsilon t}+\int_{0}^{t} e^{-\epsilon(t-s)} \mathrm{d} W_{s} \\
& =x_{0}(1-\epsilon t)+\int_{0}^{t}(1-\epsilon(t-s)) \mathrm{d} W_{s}+O\left(\epsilon^{2}\right) \\
& =x_{0}+W_{t}+\epsilon\left(-x_{0} t-\left[(t-s) W_{s}\right]_{0}^{t}-\int_{0}^{t} W_{s} \mathrm{~d} s\right)+O\left(\epsilon^{2}\right) ;
\end{aligned}
$$

in this calculation we have a conventional expansion in the second line, and we have integrated by parts; the endpoint contributions vanish on using $W_{0}=0$. Of course, the same sample path for the Brownian motion must be used throughout. It should be noted that the expansion is only valid for $t \ll O(1 / \epsilon)$, as higher order terms become comparable with those considered above when $t=O(1 / \epsilon)$. A sample simulation is shown in Figure 4. 


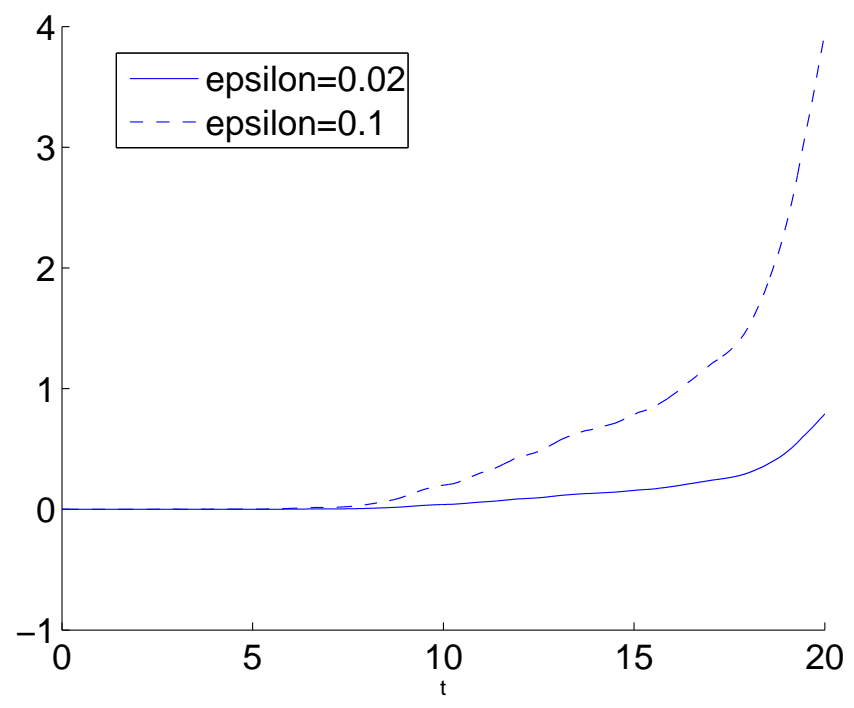

Figure 5: Difference between numerical simulations of the nonlinear process $\mathrm{d} X_{t}=-\epsilon X_{t}^{2} \mathrm{~d} t+\mathrm{d} W_{t}, \epsilon=0.02$ and $\epsilon=0.1$, and of their two-term asymptotic approximations. Divergence clearly becomes significant when $\epsilon t=O(1)$ and increases with $\epsilon$. Explicit forward scheme with timestep 0.004.

Although this example is trivial, the same technique can also be applied to problems such as

$$
\mathrm{d} X_{t}^{\epsilon}=-\epsilon\left(X_{t}^{\epsilon}\right)^{2} \mathrm{~d} t+\mathrm{d} W_{t}, \quad X_{0}=x_{0},
$$

for which the exact solution is less straightforward; however, taking $x_{0}=0$ for simplicity, successive approximation readily gives

$$
X_{t}^{\epsilon} \stackrel{P}{\sim} W_{t}-\epsilon \int_{0}^{t} W_{s}^{2} \mathrm{~d} s+O\left(\epsilon^{2}\right) ;
$$

the last term can be integrated by parts to give

$$
X_{t}^{\epsilon} \stackrel{P}{\sim} W_{t}+\epsilon\left(\frac{1}{2} t^{2}-t W_{t}^{2}+2 \int_{0}^{t} s W_{s} \mathrm{~d} W_{s}\right)+O\left(\epsilon^{2}\right) .
$$

A numerical simulation of the error is shown in Figure 5.

For a simple example of an asymptotic approximation valid in distribution, we could consider the $\mathrm{O}-\mathrm{U}$ process

$$
\mathrm{d} X_{t}^{\epsilon}=-\frac{1+\epsilon}{\epsilon} X_{t}^{\epsilon} \mathrm{d} t+\frac{1}{\sqrt{\epsilon}} \mathrm{d} W_{t}, \quad X_{0}^{\epsilon}=x_{0},
$$


for which if $t=O(1)$ the distribution is approximately the stationary distribution of the leading-order process

$$
\mathrm{d} X_{t}^{(0)}=-\frac{1}{\epsilon} X_{t}^{(0)} \mathrm{d} t+\frac{1}{\sqrt{\epsilon}} \mathrm{d} W_{t},
$$

all information about the initial value $x_{0}$ (and hence about the path) being exponentially small and hence invisible at this order. It can be seen for how long this information is significant by making the time-change $t=\epsilon \tau$, giving

$$
\mathrm{d} X_{\tau}^{\epsilon}=-(1+\epsilon) X_{\tau} d \tau+\mathrm{d} W_{\tau}
$$

(note the effect of changing the timescale on the Brownian motion $W$ ), and a regular expansion can be made, although as noted above it becomes invalid for $\tau=O(1 / \epsilon)$, at which time the initial information has effectively been forgotten.

It remains to consider the analogue of conventional matching. Bearing in mind the examples above, we formulate a tentative conjecture: matching à la Van Dyke corresponds to moving from a pathwise expansion to a distributional one. That is, specific information about the path is lost by a matching process, but distributional information is preserved. Thus, in the $\mathrm{O}-\mathrm{U}$ example just cited, the pathwise information in the 'inner layer' $t=O(\epsilon)$ is lost, but the limiting distribution for large $\tau$ of the 'inner' process $X_{\tau}$ is, to leading order, identical with the leading-order 'outer' stationary distribution. Whether this formulation is reasonable and the conjecture correct and, if so, whether the idea can give new information about other problems such as discretely sampled barrier options or stochastic processes other than Wiener processes, are all interesting questions for future research.

\section{Conclusion}

This paper deals with a small number of the many possible applications of matched asymptotic expansions in finance. As mentioned earlier, this is just one example of the kind of technology transfer that has enabled the subject of mathematical finance to develop from its infancy. It would be fair to say that, in the early days of the subject, this transfer was mostly from established areas of mathematics (probability, partial differential equations) to finance, with the primary novelty being in the modelling of financial contracts. Now, however, one may reasonably say that the level of mathematical difficulty inherent in financial models has risen to match that of the modelling: in a sense, the easy mathematical pickings are over. Current models are characterised, for example, by complexity, as they attempt to deal with more realistic problems than single options; by mathematical technicalities and difficulties such as those that arise when Levy processes are used to model asset prices, or when inverse problems are solved to determine model parameters, or when nonlinearities arise as in models of illiquid markets; by large scale of computation, as banks may have to value thousands of contracts to determine their overall mark-to-market position; and most of all by the difficulties of modelling an ever-changing market 
environment. Although the subject is 'mature', it still presents many challenges to the mathematician and financial engineer alike.

\section{Acknowledgements}

I am grateful to Joe Conlon, Ben Hambly and Michael Mitton for helpful comments, and to Mike Giles for assistance with computation.

\section{References}

[1] M.J. Lighthill, Newer Uses of Mathematics. Penguin books (1978), 432 pp.

[2] M.J. Lighthill, Introduction to Fourier Analysis and Generalised Functions. Cambridge University Press (1958), 79 pp.

[3] F. Black and M. Scholes, The pricing of options and corporate liabilities. Journal of Political Economy, 81 (1973) 637-654.

[4] R.C. Merton, Theory of rational option pricing. Bell J. Econ. Manag. Sci. 4 (1973) 141-83.

[5] Paul Wilmott, Sam Howison and Jeff Dewynne, The Mathematics of Financial Derivatives. Cambridge University Press (1995), 316 pp.

[6] M. Widdicks, P. Duck, A. Andricopoulos and D.P.Newton, The BlackScholes equation revisited: asymptotic expansions and singular perturbations. Mathematical Finance 15 (2005) 373-391.

[7] R. Almgren and N. Chriss, Optimal execution of portfolio transactions. Journal of Risk 3 (2001) 5-39.

[8] D. Bakstein and S.D. Howison, An arbitrage-free liquidity model with observable parameters for derivatives, working paper, Mathematical Institute, Oxford University (2004).

[9] R. Frey and A. Stremme, Market volatility and feedback effects from dynamic hedging. University of Bonn, Discussion Paper (1995).

[10] V. Putyatin and J. Dewynne, Market liquidity and its effects on option valuation and hedging. Phil. Trans. Roy. Soc. 357 (1999) 2093-2108.

[11] P. Schönbucher and P. Wilmott, The Feedback effect of hedging in illiquid markets. SIAM Journal of Applied Mathematics, 61 (2000) 232-272.

[12] R. Sircar and G. Papanicolaou, General Black-Scholes models accounting for increased market volatility from hedging strategies. Applied Mathematical Finance, 5 (1998) 45-82. 
[13] M. Mitton, Derivative pricing in an illiquid market. Transfer Thesis, OCIAM, Mathematical Institute, Oxford University (2003).

[14] J.R. King and C.P. Please, Diffusion of dopant in crystalline silicon: an asymptotic analysis. I.M.A. J. Appl. Maths 37 (1986) 185-197.

[15] J.P.Fouque, G. Papanicolaou and K.R. Sircar, Derivatives in Financial Markets with Stochastic Volatility, Cambridge University Press (2000), 197 pp.

[16] S.L. Heston, A closed-form solution for options with stochastic volatility with applications to bond and currency options. Review of Financial Studies, 6 (1993) 237-343.

[17] S.D. Howison, A. Rafailidis and H. Rasmussen, On the pricing and hedging of volatility derivatives. Applied Math. Finance 11 (2004) 317-346.

[18] H.O. Rasmussen and P. Wilmott, Asymptotic Analysis of Stochastic Volatility Models. In "New Directions in Mathematical Finance", Eds. P. Wilmott and H. O. Rasmussen, Wiley (2002).

[19] J.P. Fouque, G. Papanicolaou, K.R. Sircar and K. Solna, Singular perturbations in option pricing, SIAM J Appl Math 63 (2003), 1648-1665.

[20] J.G. Conlon and M.G. Sullivan, Convergence to Black-Scholes for ergodic volatility models, Preprint (2005).

[21] Howison, S.D. Practical Applied Mathematics, Cambridge University Press (2005), 326 pp.

[22] S.D. Howison and J.R. King, Ray methods for free boundary problems. Quart. Appl. Math. in press (2005).

[23] S.D. Howison, S.D. and M. Steinberg, A matched asymptotic expansions approach to continuity corrections for discretely sampled options. Part 1: barrier options. Preprint (2005). 